\title{
Membrane in M5-brane Background
}

\author{
Wei-shui $\mathrm{Xu}^{a}$ and Ding-fang Zeng ${ }^{b}$ \\ ${ }^{a}$ Institute of Theoretical Physics \\ P.O. Box 2735, Beijing 100080, P. R. China \\ ${ }^{b}$ College of Applied Science, Beijing University Of Technology \\ e-mail: wsxu@itp.ac.cn,dfzeng@bjut.edu.cn
}

\begin{abstract}
In this paper, we investigate the properties of a membrane in the M5-brane background. Through solving the classical equations of motion of the membrane, we can understand the classical dynamics of the membrane in this background.
\end{abstract}

April 2007 


\section{Introduction}

In eleven-dimensional $\mathrm{M}$ theory, there exists two extended brane solutions, i.e membrane and M5-brane. The membrane was recovered in [1] as an elementary solution of $D=11$ supergravity which preserves half of the spacetime supersymmetry, which is a electric source of four-form field. While, the M5-brane was found in [2] as a soliton solution of $D=11$ supergravity also preserving half of the spacetime supersymmetry, but is magnetic source of the same four-form field. These extended brane solutions can be related to the corresponding brane solutions in ten-dimensional string theory. After performing the compactification and some dualities, these branes can be reduced to D-branes or other brane solutions in string theory [3].

In this paper, we will investigate the properties of M2-brane in the M5-brane background. Here, we will not investigate the cases of the brane intersection. Instead, we are mainly concerned with the classical dynamics of membrane in the given background. As will be illustrated, due to the gravity force of M5-brane, the membrane evolves nontrivially.

In the 11-dimensional supergravity, the classical solution of $N$ coincident M5-brane reads

$$
\begin{aligned}
d s^{2} & =H^{-\frac{1}{3}} \eta_{\mu \nu} d x^{\mu} d x^{\nu}+H^{\frac{2}{3}} \delta_{i j} d x^{i} d x^{j} \\
H & =1+\frac{\pi N l_{p}^{3}}{R^{3}} \\
R^{2} & =\sum_{i}\left(x^{i}\right)^{2}=r^{2}+x_{11}^{2}, \quad \mu, \nu=0,1, \cdots, 5, \quad i, j=6,7,8,9,11
\end{aligned}
$$

and the 4-form field strength takes the form

$$
F_{4}=d A_{3}=3 \pi N l_{p}^{3} d v_{S^{4}}
$$

where the $d v_{S^{4}}$ denotes the volume form of a unit $S^{4}$ and $l_{p}$ is the Planck length in the 11-dimensional theory. The $N$ coincident M5-brane are parallel to the $x^{\mu}$ directions and located at $R=0$ in the transverse space. In the near horizon limit $R \rightarrow 0$, the harmonic

form $H$ will become $H=\frac{\pi N l_{p}^{3}}{R^{3}}$, and the other parts will choose the same forms as in the equations (1.1) and (1.2).

As in [4], if we suppose that there are a periodic configuration of $N$ coincident M5brane along the $x^{11}$ direction at intervals of $2 \pi R_{11}$, and take the limit of $1 \ll r / R_{11}$, then 
our background metric and the 4-form field strength will become

$$
\begin{aligned}
d s^{2} & =f^{-\frac{1}{3}} \eta_{\mu \nu} d x^{\mu} d x^{\nu}+f^{\frac{2}{3}} \delta_{i j} d x^{i} d x^{j}+f^{\frac{2}{3}}\left(d x^{11}\right)^{2} \\
f & =1+\frac{N \ell_{p}^{3}}{R_{11} r^{2}} \\
F_{4} & =\frac{2 N \ell_{p}^{3}}{R_{11}} d v_{S^{3}} \wedge d x^{11} \\
r^{2} & =\sum_{i}\left(x^{i}\right)^{2}, \quad x^{11}=R_{11} \phi
\end{aligned}
$$

where $\mu, \nu=0,1, \cdots, 5, i, j=6,7,8,9$ and $0 \leq \phi \leq 2 \pi$. We can see this metric has an so(4) symmetry group of rotations in the directions transverse to the M5-brane. In the near horizon limit, the harmonic function $f$ becomes $f=\frac{N \ell_{p}^{3}}{R_{11} r^{2}}$. While, the other parts of background (1.3) remain unchanged. Actually, if letting the radius of $x^{11}$ coordinate approach zero, then the metric (1.3) can reduce to the $N$ coincident NS5-brane solution in ten-dimensional string theory [5].

Here we will mainly focus on the classical dynamics of a M2-brane in the above backgrounds (1.1) and (1.3). The dynamics of this single membrane can be described by the Nambu-Goto and Wess-Zumino type effective action. However, for the coincident membranes, unlike the coincident D-brane in string theory which can be described by the effective action [6], their worldvolume action is still not very clear [7]. We choose the worldvolume coordinates of membrane as $x^{0}, x^{1}, x^{2}$, and those of M5-brane as $x^{0}, \cdots, x^{5}$. Hence M2-brane is "parallel" to the M5-brane, i.e it is extended in some of the M5-brane worldvolume directions $x^{\mu}$, and point-like in the directions transverse to the M5-brane $\left(x^{6}, x^{7}, x^{8}, x^{9}, x^{11}\right)$. Indeed, this configuration breaks supersymmetry completely. We can label the worldvolume coordinates of the M2-brane by $\xi^{\mu}, \mu=0,1,2$, and use reparameterization invariance on the worldvolume of the M2-brane to set $\xi^{\mu}=x^{\mu}$. The position of the M2-brane in the transverse directions, $\left(x^{6}, \cdots, x^{9}, x^{11}\right)$, give rise to scalar fields on the worldvolume of the M2-brane, $\left(X^{6}\left(\xi^{\mu}\right), \cdots, X^{9}\left(\xi^{\mu}\right), X^{11}\left(\xi^{\mu}\right)\right)$. A single M2-brane worldvolume action [8] is given by the sum of the Nambu-Goto action and the Wess-Zumino type term in the following form

$$
S_{M 2}=-T_{2} \int d^{3} \xi \sqrt{-\operatorname{det} P[G]_{\mu \nu}}+T_{2} \int P[A]
$$

where the tension of the M2-brane is expressed as $T_{2}=1 / 4 \pi^{2} l_{p}^{3}$, and $P[\cdots]$ means the pullback operation

$$
P[G]_{\mu \nu}=\frac{\partial X^{M}}{\partial \xi^{\mu}} \frac{\partial X^{N}}{\partial \xi^{\nu}} G_{M N}(X)
$$




$$
P[A]=\frac{1}{6} \epsilon^{\mu \nu \rho} \frac{\partial X^{M}}{\partial \xi^{\mu}} \frac{\partial X^{N}}{\partial \xi^{\nu}} \frac{\partial X^{L}}{\partial \xi^{\rho}} A_{M N L}(X) .
$$

The indices $M, N, L$ run over the whole eleven dimensional spacetime. And the fields $G_{M N}, A_{M N L}$ denote the metric and form field in eleven dimensions. In the following sections, we will discuss the M2-brane classical dynamics in the above backgrounds, and suppose that the transverse coordinates of M5-brane only depend on the time coordinate. In this case the Wess-Zumino term in the membrane action will vanish.

\section{Classical dynamics of membrane}

Now let us consider the membrane dynamics in the background (1.1). Since we have supposed that the directions transverse to the M5-brane $X^{i}$ are only the function of time $t$, where $i=6,7,8,9,11$, the pullback quantities are as following

$$
\begin{aligned}
P[G]_{t t} & =-H^{-\frac{1}{3}}+H^{\frac{2}{3}} \dot{X}^{i} \dot{X}^{i}, \\
P[G]_{x^{1} x^{1}} & =H^{-\frac{1}{3}}, \\
P[G]_{x^{2} x^{2}} & =H^{-\frac{1}{3}}, \quad P[A]=0 .
\end{aligned}
$$

after substituting the above equations (2.1) into the M2-brane action (1.4), we get

$$
S_{M 2}=-V T_{2} \int d t \sqrt{H^{-1}-\dot{X}^{i} \dot{X}^{i}}
$$

where $V$ is the space volume of the M2-brane, also $i=6, \cdots, 9,11$. We can find it is very similar to the corresponding one in [9] which is the DBI action of D-brane in the N NS5 brane background. Then through using the Legendre transformation, the Hamiltonian is

$$
\mathcal{H}=\frac{T_{2} V}{H \sqrt{H^{-1}-\dot{X}^{i} \dot{X}^{i}}} \equiv V E
$$

where the $E$ denotes the energy density. And the equation of motion will be

$$
\frac{d}{d t}\left(\frac{\dot{X}^{i}}{\sqrt{H^{-1}-\dot{X}^{j} \dot{X}^{j}}}\right)=\frac{\partial_{i} H}{2 H^{2} \sqrt{H^{-1}-\dot{X}^{j} \dot{X}^{j}}} .
$$

Using this equation of motion (2.4), one can check that the Hamiltonian is conserved. To

solve the (2.4), we need the initial conditions that it is $\vec{X}(t=0)$ and $\dot{\vec{X}}(t=0)$. These two vectors define a plane in $R^{5}$. By an $S O(5)$ rotation, we can rotate this plane into 
the $\left(x^{6}, x^{7}\right)$ plane. Then the motion will remain in the $\left(x^{6}, x^{7}\right)$ space for all time. Thus, without loss of generality, we can study trajectories in this space.

We choose the polar coordinates

$$
\begin{gathered}
X^{6}=R \cos \theta, \\
X^{7}=R \sin \theta .
\end{gathered}
$$

Then the energy density (2.3) will become

$$
E=\frac{T_{2}}{H \sqrt{H^{-1}-\dot{R}^{2}-R^{2} \dot{\theta}^{2}}},
$$

and the angular momentum density will be

$$
L_{\theta}=\frac{T_{2} R^{2} \dot{\theta}}{\sqrt{H^{-1}-\dot{R}^{2}-R^{2} \dot{\theta}^{2}}} .
$$

We can find this angular momentum of the M2-brane is conserved as well. From the membrane action (2.2), we can obtain energy momentum tensor. The components of $T_{\mu \nu}$ are listed in the following

$$
\begin{aligned}
T_{00} & =-\frac{T_{2}}{H \sqrt{H^{-1}-\dot{X}^{i} \dot{X}^{i}}}, \\
T_{i j} & =-T_{2} \delta_{i j} \sqrt{H^{-1}-\dot{X}^{i} \dot{X}^{i}}
\end{aligned}
$$

and the other components of stress tensor are zero. From the angular momentum $L_{\theta}$ equation (2.7) and energy density $E$ (2.6), we can get the equations of the coordinates $R$ and $\theta$

$$
\begin{gathered}
\dot{R}^{2}=\frac{1}{H}-\frac{1}{E^{2} H^{2}}\left(T_{2}^{2}+\frac{L_{\theta}^{2}}{R^{2}}\right), \\
\dot{\theta}=\frac{L_{\theta}}{E H(R) R^{2}} .
\end{gathered}
$$

For simplicity, we can first consider $L_{\theta}=0$ case, then the radial equation is

$$
\dot{R}^{2}=\frac{1}{H}-\frac{T_{2}^{2}}{E^{2} H^{2}}
$$

The right hand of the above equation can't be smaller than zero, so we get a constraint on the coordinate $R$ is

$$
\frac{\pi N l_{p}^{3}}{R^{3}} \geq \frac{T_{2}^{2}}{E^{2}}-1
$$


From the above equation, we can see if the energy density $E$ is larger than the tension of a M2-brane, $T_{2}$, the constraint $(2.12)$ is empty and the M2-brane can escape to infinity. However, for $E<T_{2}$, the M2-brane does not have enough energy to overcome the gravitational pull of the $M 5$-brane, and then will fall down to the M5-brane from an initial position.

Choosing the near horizon limit, hence the harmonic function becomes $H=\pi N l_{p}^{3} / R^{3}$. Then the equation (2.11) will be

$$
\dot{R}^{2}=\frac{1}{\pi N l_{p}^{3}} R^{3}-\frac{T_{2}^{2}}{\pi^{2} N^{2} E^{2} l_{p}^{6}} R^{6} .
$$

Since the left hand of the equation (2.13) is nonnegative, the coordinate $R$ has a maximal value $\left(\pi N E^{2} l_{p}^{3} / T_{2}^{2}\right)^{1 / 3}$. Also from this equation, the minimal value of $R$ is zero. Except for these two, there are no other extremum. But there is one inflexion between points $R=0$ and $\left(\pi N E^{2} l_{p}^{3} / T_{2}^{2}\right)^{1 / 3}$. We can regard the M2-brane is at the maximal value $\left(\pi N E^{2} l_{p}^{3} / T_{2}^{2}\right)^{1 / 3}$ at the initial time. Due to the gravitational force of M5-brane, the M2brane then will roll down to the M5-brane. As the time $t \rightarrow \infty$, the radial coordinate $\mathrm{R}$ approaches to zero. We can calculate the energy momentum tensor which is $T_{i j}=$ $-\delta_{i j} \frac{T_{2}^{2}}{E H(R)}$ as the $R \rightarrow 0$, the $T_{i j}$ will approach to zero. It may regard as the pressure decreasing to zero. But we need to mention that the coordinate $R$ can't reach zero, since at this point the supergravity background will be not reliable. Then the classical dynamics of the membrane near $R=0$ from the above analysis will become incorrect. Thus, in order to use the supergravity approximation, we must constrain the coordinate $R$ to be larger than the planck length $l_{p}$.

Now we begin to consider the nonzero case of angular momentum. From the radial equation of motion (2.9) , and after substituting the harmonic function $H=1+\pi N l_{p}^{3} / R^{3}$, we can get the constraint on the radial coordinate $R$ is

$$
\frac{1}{R^{3}}-\frac{L_{\theta}^{2}}{\pi N E^{2} l_{p}^{3}} \frac{1}{R^{2}} \geq \frac{1}{\pi N l_{p}^{3}}\left(\frac{T_{2}^{2}}{E^{2}}-1\right) .
$$

If choosing the equal case of the above equation, the constraint will become

$$
\frac{1}{R^{3}}-\frac{L_{\theta}^{2}}{\pi N E^{2} l_{p}^{3}} \frac{1}{R^{2}}-\frac{1}{\pi N l_{p}^{3}}\left(\frac{T_{2}^{2}}{E^{2}}-1\right)=0 .
$$

The above equation only has one real root which is the maximal distance that M2-brane is separated from M5-brane. 
For simplicity, we choose the near horizon limit, then the equation of motion for the radial coordinate will become

$$
\dot{R}^{2}=\frac{1}{\pi N l_{p}^{3}} R^{3}-\frac{L_{\theta}^{2}}{\pi^{2} N^{2} E^{2} l_{p}^{6}} R^{4}-\frac{T_{2}^{2}}{\pi^{2} N^{2} E^{2} l_{p}^{6}} R^{6} .
$$

We find that the equation $(\underline{2.16})$ is still very difficult to solve. Instead, here, we take some analysis for this equation. If letting $L_{\theta}=0$, then this equation will reduce to the equation (2.13). We let the left hand of the equation (2.16) to zero, then we can get the extremal value for $R$. Actually, there are two only two real extremal values of the radial coordinate $R$. One is $R=0$, the other is

$$
R=\frac{\left(108 \pi N l_{p}^{3} E^{2} T_{2}+12 \sqrt{3} \sqrt{4 L_{\theta}{ }^{6}+27 \pi^{2} N^{2} l_{p}{ }^{6} E^{4} T_{2}^{2}}\right)^{2 / 3}-12 L_{\theta}{ }^{2}}{6 T_{2}\left(108 \pi N l_{p}^{3} E^{2} T_{2}+12 \sqrt{3} \sqrt{4 L_{\theta}{ }^{6}+27 \pi^{2} N^{2} l_{p}{ }^{6} E^{4} T_{2}^{2}}\right)^{1 / 3}}
$$

When $L_{\theta}=0$, the above $R$ value will reach the $\left(\pi N E^{2} l_{p}^{3} / T_{2}^{2}\right)^{1 / 3}$. As the same in the $L_{\theta}=0$ case, between the $R=0$ and (2.17) there exists a inflexion. We can suppose that the M2-brane is at the maximal value (2.17) at the initial time, then under the gravitational pull of M5-brane, it will monotonic approach to M5-brane. Of course for the $L_{\theta}$ nonzero case, the equation (2.10) for the $\theta$ coordinate in the near horizon background is $\dot{\theta}=\frac{L_{\theta}}{E H R^{2}}=\frac{R L_{\theta}}{\pi N E l_{p}^{3}}$. Thus, if the radial coordinate $R$ reaches the value (2.17), the angular velocity will choose the maximum, and as the $R \rightarrow 0$, the angular velocity does also approach to zero. The energy momentum tensor satisfies $T_{i j}=-\delta_{i j} \frac{T_{2}^{2}}{E H(R)}=-\delta_{i j} \frac{T_{2}^{2}}{\pi N E l_{p}^{3}} R^{3}$. Thus, it again goes to zero as in the $L_{\theta}=0$ case. As mentioned in the above, near the region $R=0$, the classical background will be instability due to the strong interaction. Hence the above supergravity analysis will become unreliable in this region.

From the first section, we already know that, after compactifying a periodic circle of coordinate $x^{11}$, the metric (1.1) will become background (1.3). In the following, we study the membrane dynamics in this background (1.3). Here, we still suppose the directions transverse to the M5-brane $X^{i}$ and $X^{11}$ are only the function of time $t$, where $i=6,7,8,9$, then the pullback quantities take the form as follows

$$
\begin{aligned}
P[G]_{t t} & =-f^{-\frac{1}{3}}+f^{\frac{2}{3}} \dot{X}^{i} \dot{X}^{i}+f^{\frac{2}{3}} \dot{X}^{11} \dot{X}^{11}, \\
P[G]_{x^{1} x^{1}} & =f^{-\frac{1}{3}} \\
P[G]_{x^{2} x^{2}} & =f^{-\frac{1}{3}}, \quad P[A]=0 .
\end{aligned}
$$


After inserting (2.18) into the M2-brane action (1.4), we can get

$$
S_{M 2}=-V T_{2} \int d t \sqrt{f^{-1}-\dot{X}^{i} \dot{X}^{i}-\dot{X}^{11} \dot{X}^{11}}
$$

where $V$ is the space volume of the M2-brane. This action is also very similar to action in [9] except for the harmonic function and dimension. From the Lagrangian (2.19), we can derive the equations of motion for the membrane in this background as followes

$$
\begin{gathered}
\frac{d}{d t}\left(\frac{\dot{X}^{i}}{\sqrt{f^{-1}-\dot{X}^{j} \dot{X}^{j}-R_{11}^{2} \dot{\phi}^{2}}}\right)=\frac{\partial_{i} f}{2 f^{2} \sqrt{f^{-1}-\dot{X}^{j} \dot{X}^{j}-R_{11}^{2} \dot{\phi}^{2}}} \\
\frac{d}{d t}\left(\frac{R_{11} \dot{\phi}}{\sqrt{f^{-1}-\dot{X}^{j} \dot{X}^{j}-R_{11}^{2} \dot{\phi}^{2}}}\right)=0 .
\end{gathered}
$$

Due to some symmetry of this system, there are also some conserved charges. Time translation invariance implies that the energy

$$
\mathcal{H}=P_{i} \dot{X}^{i}+P_{\phi} \dot{\phi}-L
$$

is conserved. The momentum is obtained by varying the Lagrangian $L$,

$$
\begin{aligned}
& P_{i}=\frac{\delta L}{\delta \dot{X}^{i}}=\frac{T_{2} V \dot{X}_{i}}{\sqrt{f^{-1}-\dot{X}^{j} \dot{X}^{j}-R_{11}^{2} \dot{\phi}^{2}}}, \\
& P_{\phi}=\frac{\delta L}{\delta \dot{\phi}}=\frac{T_{2} V R_{11}^{2} \dot{\phi}}{\sqrt{f^{-1}-\dot{X}^{j} \dot{X}^{j}-R_{11}^{2} \dot{\phi}^{2}}} .
\end{aligned}
$$

Substituting (2.23) into (2.22), we find that the energy is given by

$$
\mathcal{H}=\frac{T_{2} V}{f \sqrt{f^{-1}-\dot{X}^{i} \dot{X}^{i}-R_{11}^{2} \dot{\phi}^{2}}} \equiv V E .
$$

And since the harmonic function $f=1+\frac{N \ell_{p}^{3}}{R_{11} r^{2}}$, then $\partial_{i} f(r)=X^{i} f^{\prime}(r) / r$, and one of the equations of motion (2.20) can be rewritten as

$$
\frac{d}{d t}\left(\frac{\dot{X}^{i}}{\sqrt{f^{-1}-\dot{X}^{j} \dot{X}^{j}-R_{11}^{2} \dot{\phi}^{2}}}\right)=\frac{X^{i} f^{\prime}}{2 r f^{2} \sqrt{f^{-1}-\dot{X}^{j} \dot{X}^{j}-R_{11}^{2} \dot{\phi}^{2}}}
$$

the other one is unchanged. 
To solve these equations, we need to specify some initial conditions for the coordinates. One condition is $\vec{X}(t=0)$ and $\dot{\vec{X}}(t=0)$. These two vectors define a plane in $R^{4}$. By an $S O(4)$ rotation symmetry, we can rotate this plane into the $\left(x^{6}, x^{7}\right)$ plane. The other one is $\phi(t=0)$ and $\dot{\phi}(t=0)$. Then the motion of the membrane will remain in the $\left(x^{6}, x^{7}, \phi\right)$ space for all time. Thus, without loss of generality, we can study trajectories in this space. In addition to the energy, the angular momentum of the M2-brane is conserved as well. It is given by

$$
L_{\theta}=\frac{1}{V}\left(X^{6} P^{7}-X^{7} P^{6}\right) .
$$

Using the expression for the momentum, (2.23), we find that

$$
L_{\theta}=T_{2} \frac{X^{6} \dot{X}^{7}-X^{7} \dot{X}^{6}}{\sqrt{f^{-1}-\dot{X}^{j} \dot{X}^{j}-R_{11}^{2} \dot{\phi}^{2}}} .
$$

Another interest quantity is the stress tensor $T_{\mu \nu}$ associated with the moving M2brane. The component $T_{00}$ denotes the energy density, so it is given by expression (2.25) for $E$, with the factor of the volume stripped off. We list the components of $T_{\mu \nu}$ in the following equations

$$
\begin{aligned}
T_{00} & =-\frac{T_{2}}{f \sqrt{f^{-1}-\dot{X}^{i} \dot{X}^{i}-R_{11}^{2} \dot{\phi}^{2}}}, \\
T_{i j} & =-T_{2} \delta_{i j} \sqrt{f^{-1}-\dot{X}^{i} \dot{X}^{i}-R_{11}^{2} \dot{\phi}^{2}}, \\
T_{\phi \phi} & =-T_{2} R_{11}^{2} \sqrt{f^{-1}-\dot{X}^{i} \dot{X}^{i}-R_{11}^{2} \dot{\phi}^{2}},
\end{aligned}
$$

and the other components of stress tensor are zero.

Due to the so(4) rotation symmetry in the transverse directions of M5-brane, it is convenient to change to the polar coordinates

$$
X^{6}=r \cos \theta, \quad X^{7}=r \sin \theta
$$

In these coordinates, the expressions of the energy density and angular momentum density becomes

$$
\begin{aligned}
& E=\frac{T_{2}}{f \sqrt{f^{-1}-\dot{r}^{2}-r^{2} \dot{\theta}^{2}-R_{11}^{2} \dot{\phi}^{2}}}, \\
& L_{\theta}=\frac{T_{2} r^{2} \dot{\theta}}{\sqrt{f^{-1}-\dot{r}^{2}-r^{2} \dot{\theta}^{2}-R_{11}^{2} \dot{\phi}^{2}}},
\end{aligned}
$$




$$
L_{\phi}=\frac{T_{2} R_{11}^{2} \dot{\phi}}{\sqrt{f^{-1}-\dot{r}^{2}-r^{2} \dot{\theta}^{2}-R_{11}^{2} \dot{\phi}^{2}}} .
$$

One can check directly that $L_{\theta}$ and $L_{\phi}$ are conserved by using the equations of motion (2.26) and (2.21).

In order to solve the equations of motion for the given energy and angular momentum densities $E, L_{\theta}$ and $L_{\phi}$, we would like to solve the equation (2.32) for $\dot{\theta}$, and then substitute this solution into the (2.31). Then the equation for the $\dot{\theta}$ is

$$
\dot{\theta}=\frac{L_{\theta}}{E f r^{2}} .
$$

Inserting it into (2.31), (2.32) and solving for $\dot{r}$, we find

$$
\dot{r}^{2}=\frac{1}{f}-\frac{1}{E^{2} f^{2}}\left(T_{2}^{2}+\frac{L_{\theta}^{2}}{r^{2}}+\frac{L_{\phi}^{2}}{R_{11}^{2}}\right) .
$$

Also we have the equation of $\dot{\phi}$

$$
\dot{\phi}=\frac{L_{\phi} \dot{\theta}}{L_{\theta} R_{11}^{2}} r^{2}=\frac{L_{\phi}}{E f R_{11}^{2}} .
$$

In the next, we would like to study the solutions of the equations of motion (2.34), (2.35) and (2.36).

Firstly, we consider the angular momentum $L_{\theta}=0$ case. Then Equation (2.34) implies that $\theta$ is constant, while the radial equation (2.35) takes the form

$$
\dot{r}^{2}=\frac{1}{f}-\frac{1}{E^{2} f^{2}}\left(T_{2}^{2}+\frac{L_{\phi}^{2}}{R_{11}^{2}}\right) .
$$

Since the right hand side of the equation(2.37) is non-negative, then we can get the condition $\frac{1}{f}-\frac{1}{E^{2} f^{2}}\left(T_{2}^{2}+\frac{L_{\phi}^{2}}{R_{11}^{2}}\right) \geq 0$. After substituting the harmonic function $f$, (1.3), into it, we find the constraint on $r$ (for fixed energy density $E$ )

$$
\frac{N l_{p}^{3}}{R_{11} r^{2}} \geq \frac{T_{e}^{2}}{E^{2}}-1
$$

where we can define the effective M2-brane tension is

$$
T_{e}^{2}=T_{2}^{2}+\frac{L_{\phi}^{2}}{R_{11}^{2}}
$$

From the equation of constraint (2.38), obviously, if the energy density $E$ is larger than the effective tension of a M2-brane, $T_{e}$, the constraint (2.38) is empty and the M2-brane 
can escape to infinity. For $E<T_{e}$, the M2-brane does not have enough energy to escape the gravitational pull of the M5-brane, which means that it cannot exceed some maximal distance from the M5-brane.

Under the near horizon limit, the harmonic function $f$ will become $f=\frac{N l_{p}^{3}}{R_{11} r^{2}}$. Then the equation (2.38) will be $\frac{N l_{p}^{3}}{R r_{1} r^{2}} \geq \frac{T_{e}^{2}}{E^{2}}$. Thus, if $r<<\sqrt{\frac{N l_{p}^{3}}{R_{11}}}$, the effective tension of membrane $T_{e}$ satisfies the constraint $T_{e} / E>>1$. However, $r>>\sqrt{\frac{N l_{p}^{3}}{R_{11}}}$, the case will be otherwise. Indeed, in this near horizon case, we can solve for the trajectory $r(t), \phi(t)$ exactly. Substituting the harmonic function $f=\frac{N l_{p}^{3}}{R_{11} r^{2}}$ into (2.37), we find the equation of motion

$$
\dot{r}^{2}=\frac{1}{N l_{p}^{3}} r^{2}-\frac{R_{11}^{2}}{E^{2} N^{2} l_{p}^{6}}\left(T_{2}^{2}+\frac{L_{\phi}^{2}}{R_{11}^{2}}\right) r^{4} .
$$

Then the solution can be obtained

$$
\frac{1}{r}=\sqrt{\frac{L_{\phi}^{2}+R_{11}^{2} T_{2}^{2}}{N R_{11} E^{2} l_{p}^{3}}} \cosh \sqrt{\frac{R_{11}}{N l_{p}^{3}}} t
$$

where we choose $t=0$ to be the time at which the M2-brane reaches its maximal distance from the M5-brane. For an observer living on M5-brane, the M2-brane reaching $r=0$ will take an infinite time. Also, the M2 radial motion is similar to D-brane's motion in [9].

And the equation of motion (2.24) becomes

$$
\dot{\phi}^{2}=\frac{\frac{R_{11} L_{\phi}^{2}}{N l_{p}^{3}} r^{2}-\dot{r}^{2}}{L_{\phi}^{2} R_{11}^{2}+T_{2}^{2} R_{11}^{4}} .
$$

Substituting the solution $r$ into equation (2.42), we can get the equation

$$
\dot{\phi}=\frac{L_{\phi} E}{L_{\phi}^{2}+T_{2}^{2} R_{11}^{2}}\left(\cosh \sqrt{\frac{R_{11}}{N l_{p}^{3}}} t\right)^{-2} .
$$

Then after solving this equation, the solution can be obtained

$$
\phi=\frac{\sqrt{\frac{N l_{p}^{3}}{R_{11}}} L_{\phi} E}{L_{\phi}^{2}+T_{2}^{2} R_{11}^{2}} \tanh \sqrt{\frac{R_{11}}{N l_{p}^{3}}} t .
$$

It is interesting to calculate the energy momentum tensor of the M2-brane in this case. The energy density $T_{00}$ is constant and equal to $E$ throughout the time evolution. 
However, for the parts $T_{i j}$ and $T_{\phi \phi}$, we can find

$$
\begin{aligned}
T_{i j} & =-\delta_{i j} \frac{T_{2}^{2}}{E f}, \\
T_{\phi \phi} & =-\frac{R_{11}^{2} T_{2}^{2}}{E f} .
\end{aligned}
$$

We see that the pressure goes smoothly to zero as $r \rightarrow 0$, since $f(r) \sim 1 / r^{2}$. But again as the analysis in the background (1.1), this may be unreliable near the $r=0$ region.

So far we have discussed the trajectories with vanishing angular momentum density (2.32). A natural question is whether anything qualitatively new occurs for non-zero $L_{\theta}$. Just as [9], we can think as follows, the radial equation of motion (2.35) can be thought of as describing a particle with mass $m=2$, moving in one dimension $r$ in the effective potential

$$
V_{\mathrm{eff}}(r)=\frac{1}{E^{2} f^{2}}\left(T_{2}^{2}+\frac{L_{\theta}^{2}}{r^{2}}+\frac{L_{\phi}^{2}}{R_{11}^{2}}\right)-\frac{1}{f}
$$

with zero energy. Now we discuss the properties of this effective potential $V_{\text {eff }}$. In the small $r$ region, it will behave as

$$
V_{\mathrm{eff}}(r) \simeq \frac{R_{11}}{N l_{p}^{3}}\left(\frac{R_{11} L_{\theta}^{2}}{E^{2} N l_{p}^{3}}-1\right) r^{2} .
$$

For large $r$, the leading terms of this potential will be

$$
V_{\mathrm{eff}}(r) \simeq \frac{T_{e}^{2}}{E^{2}}-1
$$

If the energy density of the M2-brane is smaller than the effective tension of a M2-brane, $E<T_{e}$, then the effective potential $V_{\text {eff }}$ approaches to a positive constant (2.48) as $r \rightarrow \infty$, which means the membrane cannot escape to infinity. From the equation (2.47), we can find that in order to have trajectories at non-zero $r$, the angular momentum must satisfy the constraint

$$
L_{\theta}<\sqrt{\frac{N E l_{p}^{3}}{R_{11}}} .
$$

If the constraint (2.49) is not satisfied, the only solution is $r=0$. But, if the condition (2.49) is satisfied, the trajectory of the M2-brane is qualitatively similar to that in the $L_{\theta}=0$ case. It will approach the M5-brane and does not have stable orbits at finite $r$.

For the case $T_{e}>>E$, the whole trajectory lies again in the region $r<<\sqrt{N l_{p}^{3} / R_{11}}$, and one can approximate the harmonic function (1.3) by $f=\frac{N l_{p}^{3}}{R_{11} r^{2}}$. Then the equation 
(2.35) for $\dot{r}$ will be

$$
\dot{r}^{2}=\frac{R_{11}}{N l_{p}^{3}}\left(1-\frac{R_{11} L_{\theta}^{2}}{N l_{p}^{3} E^{2}}\right) r^{2}-\frac{R_{11}^{2}}{E^{2} N^{2} l_{p}^{4}}\left(T_{2}^{2}+\frac{L_{\phi}^{2}}{R_{11}^{2}}\right) r^{4},
$$

with the solution

$$
\frac{1}{r}=\sqrt{\frac{L_{\phi}^{2}+R_{11}^{2} T_{2}^{2}}{R_{11} N E^{2} l_{p}^{3}-R_{11} L_{\theta}^{2}}} \cosh \frac{\sqrt{N R_{11} E^{2} l_{p}^{3}-R_{11}^{2} L_{\theta}^{2}}}{N E l_{p}^{3}} t .
$$

We can find that the non-zero angular momentum can slow down the exponential decrease of $r$ as $t \rightarrow \infty$. In the near horizon limit $f(r)=\frac{N l_{p}^{3}}{r^{2}}$, the solution of the equation (2.34) for $\theta$ is

$$
\theta=\frac{R_{11} L_{\theta}}{E N l_{p}^{3}} t
$$

The solution (2.51) and (2.52) mean that the M2-brane in the background (1.3) will be spiralling towards the origin, circling around it an infinite number of times in the process. The equation about $\phi$ is

$$
\dot{\phi}=\frac{L_{\phi}\left(N E^{2} l_{p}^{3}-R_{11} L_{\theta}^{2}\right)}{E N l_{p}^{3}\left(L_{\phi}^{2}+R_{11}^{2} T_{2}^{2}\right)}\left(\cosh \frac{\sqrt{R_{11} E^{2} N l_{p}^{3}-R_{11}^{2} L_{\theta}^{2}}}{E N l_{p}^{3}} t\right)^{-2} .
$$

and the solution of the above equation reads

$$
\phi=\frac{L_{\phi}}{L_{\phi}^{2}+R_{11}^{2} T_{2}^{2}} \sqrt{\frac{N E^{2} l_{p}^{3}-L_{\theta}^{2}}{R_{11}}} \tanh \frac{\sqrt{R_{11} N E^{2} l_{p}^{3}-R_{11}^{2} L_{\theta}^{2}}}{E N l_{p}^{3}} t .
$$

At $t=0$, the $\phi=0$, however, the time $t \rightarrow \infty$, then, $\phi \rightarrow \frac{L_{\phi}}{L_{\phi}^{2}+R_{11}^{2} T_{2}^{2}} \sqrt{\frac{N E^{2} l_{p}^{3}-L_{\theta}^{2}}{R_{11}}}$.

Thus, the non-zero angular momentum $L_{\theta}$ slows down the variation of $\phi$. From these three solutions, we know that the M2-brane is circling along the $\theta$ direction, varying along the $\phi$ and falling down towards the M5-brane in the process. Also, the energy momentum tensor $T_{i j}$ and $T_{\phi \phi}$ will approach to zero as $r \rightarrow 0$, since $f(r) \sim 1 / r^{2}$. But we must mention that, near the $r=0$ region, the discussion may be incorrect due to the strong coupling.

In the background (1.3), the results about the dynamics of a M2-brane have some similar properties as studying in [9]. This can be understood that the D2-brane and NS5-brane in IIA can be got by compactified one transverse dimension of M2-brane and M5-brane in M theory. The solutions of equation of motion describe the M2-brane falling 
towards the M5-brane. In the non-zero angular momentum $L_{\theta}$, the M2-brane is spiralling towards the M5-brane. But both in this two case, M2-brane has a angular momentum $L_{\phi}$. We need to mention that the background (1.3) is only correct in the limit of $1 \ll r / R_{11}$. Therefore, as the M2-brane approaches the M5-brane, the energy momentum tensor $T_{i j}$ and $T_{\phi \phi}$ approaching zero may be unreliable. Since here the radial coordinate $r$ is smaller than the radius $R_{11}$. So we are not sure whether the membrane will have the same behavior just like the late time behavior of unstable D-brane [10, 11, 12, 13, 14].

In the above sections, we investigated the membrane classical dynamics in various M5-brane backgrounds. There may be some generalizations, since under the Penrose limit, the $N$ coincident M5-brane solution (1.1) will reduce to the $A d S_{7} \times S^{4}$ geometry. Hence one can investigate the membrane dynamics in this geometry. For the (1.1), (1.3) and their near horizon background geometry, after calculating the classical equations of motion of membrane from the membrane action (1.4), we can analyze the moving trajectories of membrane. In some particular cases, we can get the exact solution of trajectories of membrane. However, generally, the equations of motion is very difficult to solve. But through analyzing these equations, we still can obtain some qualitative information about the motion of membrane. Consequently, in the M5-brane background, the membrane will be falling and spiralling towards to the M5-brane by the gravitational force of M5-brane. In the near M5-brane region, i.e $R$ ( or $r$ ) being of the order of the planck length $l_{p}$, the above analysis of the classical dynamics of membrane may not be trusted, since the method of the supergravity approximation is unreliable.

\section{Acknowledgements}

We would like to thank Yi-hong Gao for the useful suggestions and discussions.

\section{References}

[1] M. J. Duff and K. Stelle, "Multimembrane solutions of d = 11 supergravity," Phys. Lett. B 253 (1991) 113.

[2] R. Gueven, "Black p-brane solutions of D = 11 supergravity theory," Phys. Lett. B 276 (1992) 49. 
[3] J. Polchinski, "String Theory (Vol. I, Vol. II)," Cambridge Press, 1998.

[4] Y. Hyakutake, "Expanded Strings in the Background of NS5-branes via a M2-brane, a D2-brane and D0-branes," hep-th/0112073.

[5] C. G. Callan, J. A. Harvey and A. Strominger, "Worldbrane actions for string solitons," Nucl. Phys. B367: 60-82, 1991; "World sheet approach to heterotic instantons and solitons," Nucl. Phys. B359: 611-634, 1991.

[6] R. C. Myers, "Dielectric branes," JHEP 9912: 022, 1999 [hep-th/9910053].

[7] A. Basu and J. A. Harvey, "The M2-M5 brane system and a generalized Nahm's equation," Nucl. Phys. B713: 136-150, 2005 [hep-th/0412310].

[8] E. Bergshoeff, E. Sezgin and P. K. Townsend, "Properties Of The Eleven-Dimensional Super Membrane Theory," Annals Phys 185 (1988) 330.

[9] D. Kutasov, "D-Brane Dynamics Near NS5-Branes," hep-th/0405058; "A Geometric interpretation of the open string tachyon," hep-th/0408073; K. L. Panigrahi, "DBrane Dynamics in Dp-Brane Background," hep-th/0407134.

[10] A. Sen, "Tachyon dynamics in open string theory," Int. J. Mod. Phys. A20: 55135656, 2005 [hep-th/0410103].

[11] A. Sen, "Rolling tachyon," JHEP 0204, 048 (2002) [hep-th/0203211].

[12] F. Larsen, A. Naqvi and S. Terashima, "Rolling tachyons and decaying branes," hep-th/0212248.

[13] T. Okuda and S. Sugimoto, "Coupling of rolling tachyon to closed strings," Nucl. Phys. B647, 101 (2002) [hep-th/0208196].

[14] N. Lambert, H. Liu and J. Maldacena, "Closed strings from decaying D-branes," hep-th/0303139. 